\title{
MÉTODOS PROBABILÍSTICO E DETERMINÍSTICO PARA DIAGNÓSTICO DA NECESSIDADE DE SUBSOLAGEM DE SOLOS AGRÍCOLAS ${ }^{1}$
}

\author{
GILMAR DA SILVA², NELSON L. CAPPELLI ${ }^{3}$, CLAUDIO K. UMEZU ${ }^{4}$
}

\begin{abstract}
RESUMO: Este trabalho apresenta duas metodologias para avaliar os níveis de resistência mecânica à penetração e auxiliar na tomada de decisão quanto à necessidade de se proceder à operação de subsolagem. A tomada de decisão baseia-se no volume de solo a ser mobilizado em função da resistência mecânica à penetração considerada crítica para o caso. $\mathrm{O}$ primeiro método, denominado probabilístico, utiliza-se de técnicas estatísticas para a definição do volume de solo a ser mobilizado. O segundo método, denominado determinístico, utiliza-se do cálculo da porcentagem do volume de solo a ser mobilizado e de sua distribuição espacial. Em ambos, são construídas curvas de porcentagem de pontos experimentais com níveis de resistência mecânica à penetração igual ou superior ao nível estabelecido como crítico, curvas de volume de solo a ser mobilizado em função do nível e, finalmente, o método determinístico apresenta ainda uma distribuição espacial dos pontos com resistência à penetração igual ou superior ao nível crítico. Uma comparação entre as curvas de volume de solo a ser mobilizado em função do nível crítico, obtidas por cada um dos métodos, mostra que os métodos são equivalentes. $\mathrm{O}$ método determinístico tem a vantagem de mostrar a distribuição espacial dos pontos críticos.
\end{abstract}

PALAVRAS-CHAVE: compactação do solo, subsolagem, resistência mecânica à penetração.

\section{PROBABILISTIC AND DETERMINISTIC METHODS FOR AGRICULTURAL SUBSOILING DIAGNOSIS}

SUMMARY: This paper presents two techniques to evaluate soil mechanical resistance to penetration as an auxiliary method to help in a decision-making in subsoiling operations. The decision is based on the volume of soil mobilized as a function of the considered critical soil resistance to penetration in each case. The first method, probabilistic, uses statistical techniques to define the volume of soil to be mobilized. The other method, deterministic, determines the percentage of soil to be mobilized and its spatial distribution. Both cases plot the percentage curves of experimental data related to the soil mechanical resistance to penetration equal or larger to the established critical level and the volume of soil to be mobilized as a function of critical level. The deterministic method plots showed the spatial distribution of the data with resistance to penetration equal or large than the critical level. The comparison between mobilized soil curves as a function of critical level using both methods showed that they can be considered equivalent. The deterministic method has the advantage of showing the spatial distribution of the critical points.

KEYWORDS: soil compactation, subsoiling, cone index.

\footnotetext{
${ }^{1}$ Parte da dissertação de Mestrado do primeiro autor. Projeto financiado pela FAPESP.

${ }^{2}$ Eng. Agrônomo, M.Sc. em Engenharia Agrícola, DMAQAG/FEAGRI/UNICAMP, Campinas - SP, Prof. M.Sc., CREUPI/PINHAL, Fone: (0XX19) 542.1707, e-mail: gsilva@agr.unicamp.br

3 Prof. Doutor, Faculdade de Engenharia Agrícola, FEAGRI/UNICAMP, Campinas - SP, Fone: (0XX19) 3788.1052, e-mail: cappelli@agr.unicamp.br

${ }^{4}$ Doutorando em Engenharia Agrícola, M.Sc. em Engenharia Elétrica, Laboratório de Instrumentação e Controle (LIC), FEAGRI/ UNICAMP, Campinas - SP, Prof. Adjunto M.Sc., Universidade São Francisco - USF, Itatiba - SP.

Recebido pelo Conselho Editorial em: 14-10-2002

Aprovado pelo Conselho Editorial em: 5-11-2003
} 


\section{INTRODUÇÃO}

O crescimento e o desenvolvimento das plantas, que as levam a produzir grãos, fibras e outros produtos comerciáveis, dependem da harmonia de uma série de fatores ambientais. A absorção de nutrientes é um dos fatores importantes para que se tenha boas produções, e qualquer obstáculo que restrinja o crescimento radicular, reduz a absorção.

Existem diversos fatores que ocasionam um crescimento deficiente do sistema radicular de plantas cultivadas, podendo ser citados: danos causados por insetos e moléstias, deficiências nutricionais, acidez do solo, drenagem insuficiente, baixa taxa de oxigênio, temperatura imprópria do solo, compactação do solo e dilaceramento radicular. Dentre essas limitações, a compactação do solo atinge, muitas vezes, dimensões sérias, pois, ao causar restrições ao crescimento e desenvolvimento radiculares, acarreta uma série de problemas que afetam a produção das plantas. Os problemas de ordem agronômica devido à compactação do solo são: aumento da resistência mecânica à penetração radicular, redução da aeração, alteração do fluxo de água e calor, e da disponibilidade de água e nutrientes.

O desenvolvimento das plantas e a produção das culturas são diretamente afetados pelas condições do solo, notadamente no que diz respeito à presença de camadas compactadas. Na cultura da aveia, EHLERS et al. (1983) verificaram que o crescimento radicular decresceu linearmente com o aumento da resistência à penetração.

Segundo TAYLOR \& BELTRAME (1980), o rompimento das camadas compactadas do solo traz como benefícios imediatos: a redução da densidade do solo, o aumento no volume dos macroporos $\mathrm{e}$, ainda, permite um fluxo vertical mais rápido da água.

TORRES \& SARAIVA (1999) afirmam que resistências à penetração, em torno de 3,5 a 6,5 MPa, aparentemente, são as mais corretas para considerar que um solo está com possíveis problemas de impedimento mecânico. Afirmam, ainda, que o ideal seria que as medidas, para quantificar a resistência do solo, fossem feitas por métodos diretos, ou seja, por meio do próprio sistema radicular. Porém esses métodos são de difícil aplicação prática, principalmente na cultura de cana-de-açúcar, na qual se cultivam milhares de hectares. Os autores concluem que os penetrômetros, apesar de suas limitações, ainda são um meio importante para avaliar a resistência mecânica à penetração no solo.

SILVA (2002) afirma que uma forma de otimizar a operação de subsolagem é realizá-la apenas nos locais e profundidades necessárias para a ruptura das camadas compactadas do solo e que isso pode ser feito dispondo-se de estudos que identifiquem a variabilidade espacial das camadas compactadas do solo, bem como de curvas de volume de solo a ser mobilizado em função da resistência mecânica à penetração considerada crítica.

O presente trabalho tem como objetivo apresentar dois métodos auxiliares, baseados na resistência mecânica à penetração, obtida por meio de penetrômetro, para a decisão de se proceder à operação de subsolagem de solos agrícolas.

\section{MATERIAL E MÉTODOS}

A área experimental escolhida para a tomada de dados foi de renovação da cultura de cana-deaçúcar, pertencente à Usina Santa Lúcia S.A., situada no município de Araras - SP (latitude $22^{\circ} 20^{\prime} 44,6^{\prime} \mathrm{S}$, longitude $47^{\circ} 25^{\prime} 00,9^{\prime} \mathrm{W}$ ). O talhão utilizado possui 12,59 ha dos quais 1,21 ha foi demarcado para o levantamento dos dados. O tipo de solo encontrado, conforme classificação da EMBRAPA (1999), foi o Nitossolo Vermelho. O talhão apresentou uma declividade média de 5\%. 
A malha amostral constituiu-se de 121 pontos de coleta de dados igualmente espaçados, cada um localizado no centro de uma grade regular de $10 \mathrm{~m}$ x $10 \mathrm{~m}$, formando uma área quadrada de $110 \mathrm{~m} \mathrm{x}$ $110 \mathrm{~m}$.

A resistência mecânica à penetração foi obtida em função da profundidade em cada um dos pontos amostrais. Para tanto, foi utilizado o penetrômetro eletrônico apresentado por CAPPELLI et al. (2001).

Para o presente estudo, foram definidas três camadas horizontais: 0 a $150 \mathrm{~mm} ; 150 \mathrm{~mm}$ a $300 \mathrm{~mm}$, e $300 \mathrm{~mm}$ a $500 \mathrm{~mm}$ de profundidade. O valor tomado como representativo de cada camada, em cada um dos 121 pontos amostrais, foi a média da resistência mecânica à penetração registrada pelo penetrômetro eletrônico nas respectivas camadas.

No primeiro método proposto, denominado probabilístico, confeccionaram-se e analisaram-se as curvas de resistência mecânica à penetração em função da profundidade, verificou-se a normalidade dos dados e analisaram-se os parâmetros estatísticos referentes à medida da tendência central e de sua variabilidade, e, finalmente, confeccionaram-se e analisaram-se as curvas de probabilidade de ocorrência de resistência mecânica à penetração igual ou superior à considerada crítica, em função do valor crítico de resistência mecânica à penetração.

O segundo método proposto, denominado determinístico, baseou-se na porcentagem do número de pontos, portanto na porcentagem do volume de solo onde ocorreram níveis de resistência mecânica à penetração acima da considerada crítica e em sua distribuição espacial, tanto no perfil horizontal quanto no vertical.

Nesse método, cada um dos pontos experimentais representou uma área e um volume de influência. O volume de solo correspondente a cada ponto foi representado por um paralelepípedo (Figura 1) cuja área da base foi formada pela área de influência de cada ponto (10 m por $10 \mathrm{~m})$ e cuja altura correspondia à espessura da camada horizontal sob estudo.

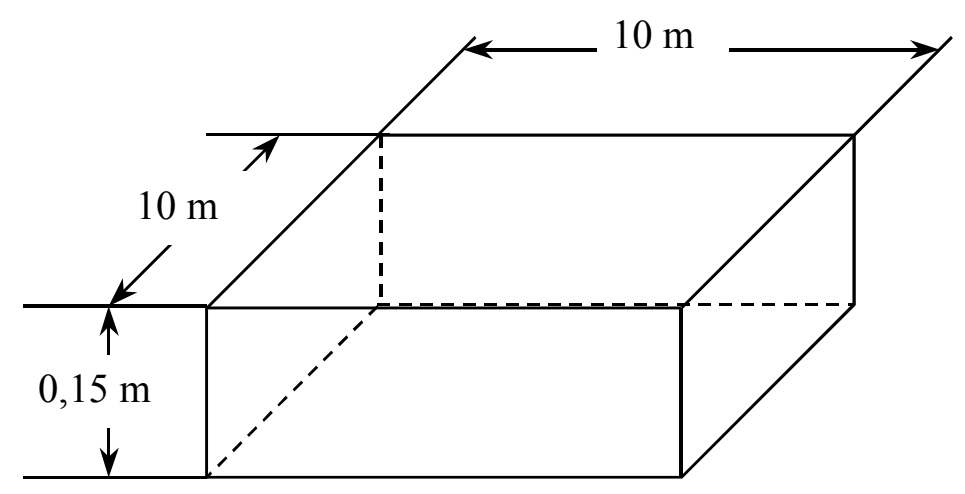

FIGURA 1. Volume de solo correspondente a cada ponto amostral.

O volume total de solo estudado $\left(6.050 \mathrm{~m}^{3}\right)$ foi considerado como o paralelepípedo cuja área da base foi formada pela área total $(110 \mathrm{~m} \times 110 \mathrm{~m})$ e cuja altura correspondia à máxima profundidade de penetração da haste do penetrômetro $(500 \mathrm{~mm})$.

Pautadas nessas definições, foram construídas curvas de porcentagem de ocorrências de resistência mecânica à penetração $(\mathrm{RP})$ igual ou superior à considerada crítica $(\mathrm{RPc})$, em função do valor considerado crítico para a resistência mecânica à penetração, para cada uma das camadas estudadas.

Em ambos os métodos, utilizou-se do "software Excel" para a realização dos cálculos e confecção dos gráficos. Com a planilha desenvolvida, foi possível fazer simulações quanto à 
localização dos pontos onde ocorreram RP igual ou superior à RPc, para cada camada estudada. Por meio da mesma planilha, foram construídas as curvas, para cada uma das camadas estudadas, de volume de solo a ser mobilizado em função do valor estabelecido como crítico para a resistência mecânica à penetração. Nessas curvas, o volume de solo a ser mobilizado foi o do paralelepípedo formado pela área de influência do ponto e pela máxima profundidade da camada estudada, já que a profundidade da operação de subsolagem compreende a espessura da própria camada, bem como a das camadas que se posicionam acima da considerada.

Finalmente, analisando-se as informações que os métodos oferecem, tais como: probabilidade de ocorrência de RP igual ou superior à RPc, porcentagem de ocorrência de pontos com níveis de RP acima do desejável, localização e distribuição espacial dos pontos críticos, bem como o volume de solo necessário de se mobilizar, pôde-se ter subsídios para estabelecer o diagnóstico quanto à necessidade, ou não, de proceder à subsolagem.

\section{RESULTADOS E DISCUSSÃO}

$\mathrm{Na}$ Figura 2, apresenta-se a posição dos pontos experimentais, bem como sua nomenclatura.

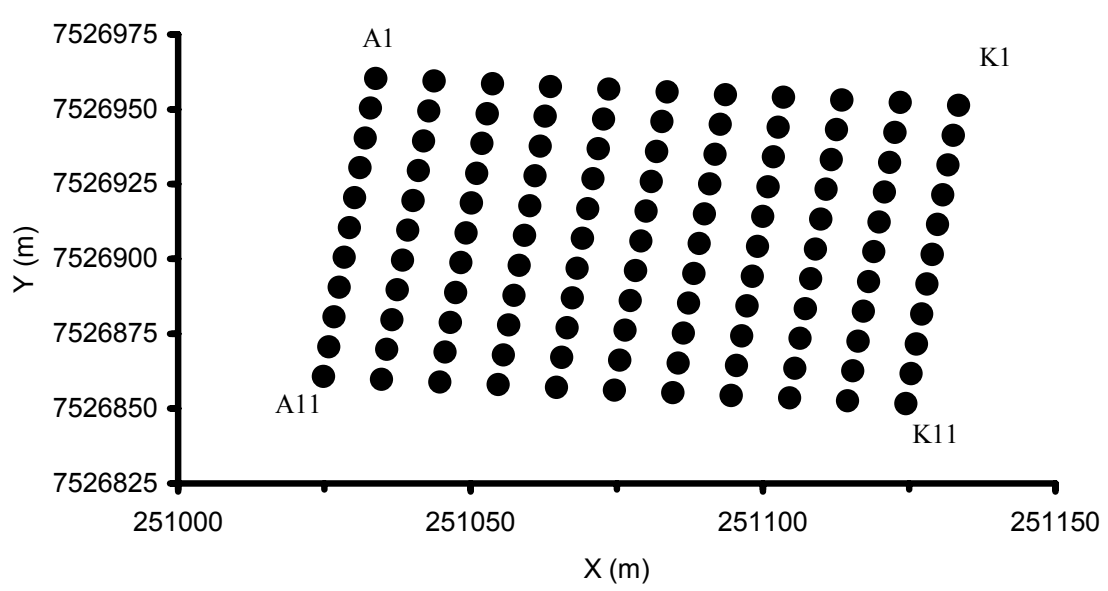

FIGURA 2. Posição dos pontos experimentais (Coordenadas UTM - WGS84).

Registrou-se o teor de água do solo para possibilitar melhor análise da resistência mecânica à penetração, bem como para ser utilizado em correções futuras, quando a pesquisa disponibilizar métodos confiáveis de correção da resistência mecânica à penetração em função do teor de água e do tipo de solo.

No mesmo dia em que foi realizada a medição da resistência mecânica à penetração, foram retiradas 36 amostras de solo para a determinação do seu teor de água, correspondendo, respectivamente, às camadas de solo de 0 a $200 \mathrm{~mm}$; de $200 \mathrm{~mm}$ a $400 \mathrm{~mm}$, e de $400 \mathrm{~mm}$ a $600 \mathrm{~mm}$ de profundidade. Os teores de água determinados representam o valor médio de cada camada considerada.

Notou-se que o teor médio de água do solo foi ligeiramente maior quanto mais profunda foi a camada estudada. No entanto, o valor absoluto do teor de água médio do solo variou pouco entre as camadas (respectivamente, $19,1 \% ; 20,4 \%$ e $20,7 \%$ ).

A variação do teor de água, obtida nas respectivas camadas, foi considerada baixa em todos os casos. Na camada de 0 a $200 \mathrm{~mm}$, o desvio-padrão foi $2,4 \%$ e o coeficiente de variação 0,13 . $\mathrm{Na}$ camada de 200 a $400 \mathrm{~mm}$, o desvio-padrão foi $1,6 \%$ e o coeficiente de variação 0,08 . Na camada de 400 a 600 mm, o desvio-padrão foi $1,9 \%$ e o coeficiente de variação 0,09 . 
Nas Figuras 3a e 3b, apresenta-se o comportamento da resistência mecânica à penetração em função da profundidade dos pontos amostrados no campo, referenciados pelas colunas B e I e pelas linhas de 1 a 11.

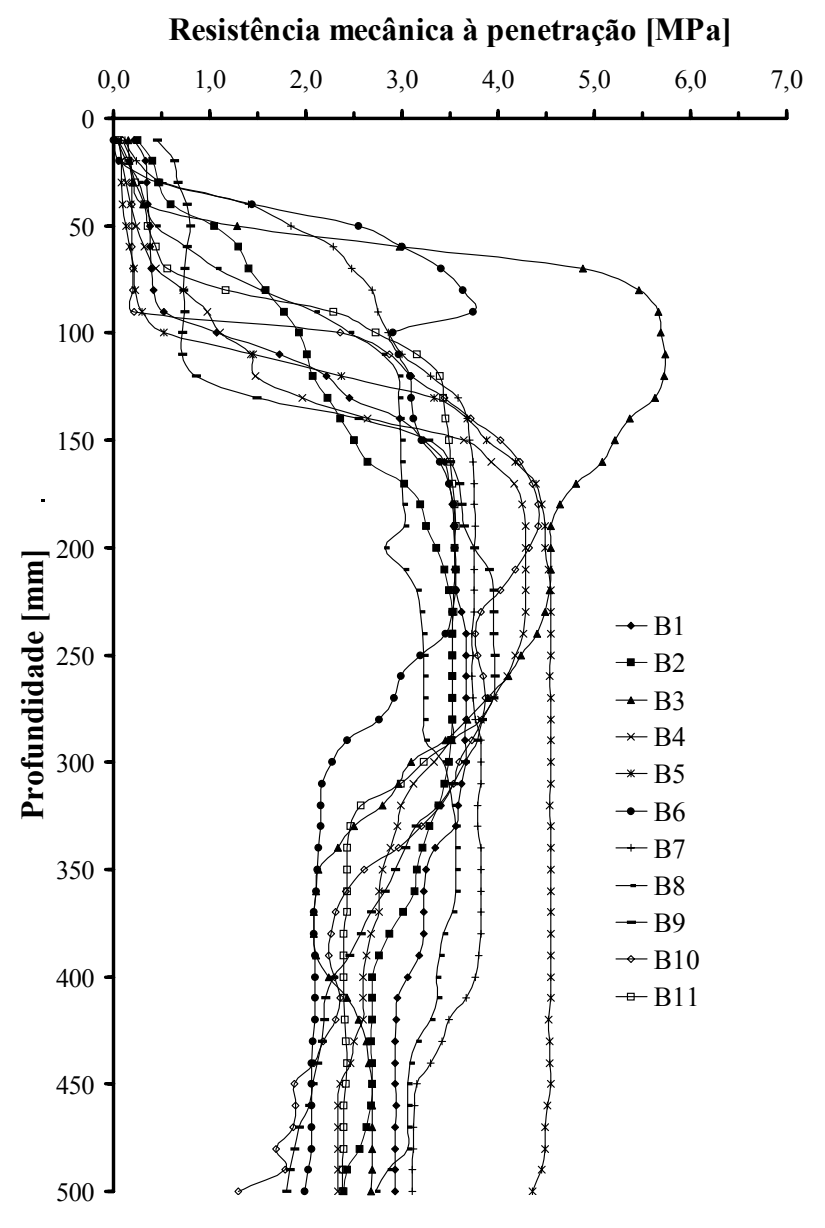

(a)

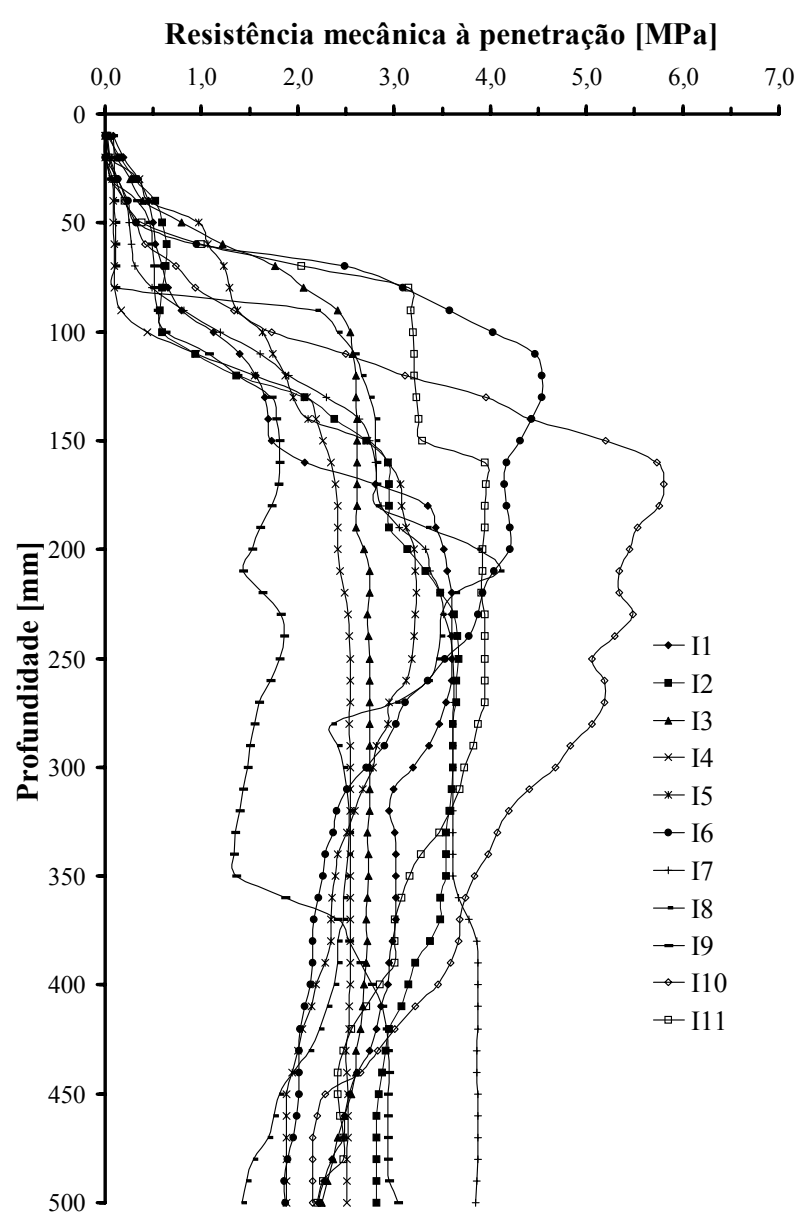

(b)

FIGURA 3. Resistência mecânica à penetração dos pontos amostrados na coluna $\mathrm{B}$ (a) e resistência mecânica à penetração dos pontos amostrados na coluna I (b).

Analisando-se as curvas de resistência mecânica à penetração em função da profundidade (Figuras 3a e 3b), verifica-se que houve um comportamento semelhante para a maioria dos pontos amostrais da coluna B e I, nas camadas de 0 a 150; de 150-300, e de 300-500 mm de profundidade. Verifica-se, também, que os maiores índices de resistência mecânica à penetração apresentaram-se no perfil horizontal intermediário, ou seja, entre 150 a $300 \mathrm{~mm}$ de profundidade. Notou-se, ainda, um comportamento crescente da resistência mecânica à penetração na camada mais superficial $(0$ a $150 \mathrm{~mm}$ ) e um comportamento tendendo a manter-se aproximadamente constante na camada mais profunda (300 a $500 \mathrm{~mm}$ ).

Antes de proceder à análise estatística dos dados, correspondente às camadas horizontais do solo, testou-se a normalidade de sua distribuição. Na Tabela 1, verifica-se que, em todos os perfis considerados, o teste de Kolmogorov-Smirnov permitiu aceitar a hipótese de que os dados vieram de uma população com distribuição normal, em um nível de significância de $1 \%$, e, portanto, as análises estatísticas, com respeito à medida da tendência central e sua variabilidade, podem ser realizadas. 
TABELA 1. Parâmetros estatísticos referentes à resistência mecânica à penetração (MPa).

\begin{tabular}{lccc}
\hline \multirow{2}{*}{ Parâmetros Estatísticos } & \multicolumn{3}{c}{ Camadas $(\mathrm{mm})$} \\
\cline { 2 - 4 } & 0 a 150 & 150 a 300 & 300 a 500 \\
\hline Número de dados & 121 & 121 & 121 \\
Valor mínimo & 0,001 & 0,483 & 1,392 \\
Valor máximo & 3,777 & 5,590 & 4,523 \\
Média & 1,466 & 3,499 & 2,811 \\
Mediana & 1,405 & 3,591 & 2,726 \\
Desvio-padrão & 0,764 & 0,889 & 0,561 \\
Coeficiente de variação & 0,521 & 0,254 & 0,200 \\
Assimetria & 0,458 & $-0,982$ & 0,492 \\
Curtose & 0,140 & 2,250 & 0,743 \\
Estatística Kolmogorov-Smirnov & 0,057 & 0,138 & 0,079 \\
Valor crítico de K-S, a 1\% & 0,147 & 0,147 & 0,147 \\
\hline
\end{tabular}

A diferença entre os valores de resistência mecânica média à penetração, obtida entre as camadas estudadas, mostra que cada perfil horizontal apresenta um comportamento distinto quanto a esse parâmetro estatístico.

A resistência mecânica média à penetração do solo foi maior na camada intermediária estudada, seguida da camada mais profunda, confirmando o que se observou, de maneira geral, nos gráficos de resistência mecânica à penetração.

Os desvios-padrão obtidos são altos em relação à média, ou seja, os coeficientes de variação obtidos são: $52,1 \% ; 25,4 \%$ e $20,0 \%$, respectivamente, para as camadas de 0 a $150 \mathrm{~mm} ; 150 \mathrm{~mm}$ a $300 \mathrm{~mm}$, e $300 \mathrm{~mm}$ a $500 \mathrm{~mm}$ de profundidade. Esse fato e a observação dos valores máximos e mínimos medidos, em todos os casos, permitem concluir que o solo apresentou grande variabilidade na resistência mecânica média à penetração em cada uma das camadas estudadas.

Nota-se que essa variabilidade diminui à medida que a profundidade aumenta, o que confirma a observação das curvas de resistência mecânica à penetração, em função da profundidade, apresentada nas Figuras 3a e 3b.

$\mathrm{Na}$ Figura 4a, apresenta-se a probabilidade de encontrar valores de resistência mecânica à penetração (RP) igual ou superior à considerada crítica (RPc) nos perfis horizontais de solos estudados.

$\mathrm{Na}$ Figura 4b, apresentam-se as curvas de volume de solo a ser mobilizado, em função dos valores críticos de resistência mecânica à penetração, nos perfis horizontais de solos estudados, obtidas pelo método probabilístico.

Observa-se que as curvas de probabilidade apresentadas na Figura 4a são do tipo "S", conforme esperado, já que os dados provêm de uma distribuição normal.

As curvas de probabilidade ratificam o comportamento anteriormente obtido da resistência mecânica à penetração para as três camadas estudadas.

Nota-se que, para a camada intermediária, a curva de probabilidade de encontrar valores de resistência mecânica à penetração igual ou superior à considerada crítica, posiciona-se mais à direita do que as outras, pois possui média de resistência mecânica à penetração superior às demais. $\mathrm{O}$ mesmo ocorre com a curva de probabilidade da camada mais profunda em relação à mais superficial. 


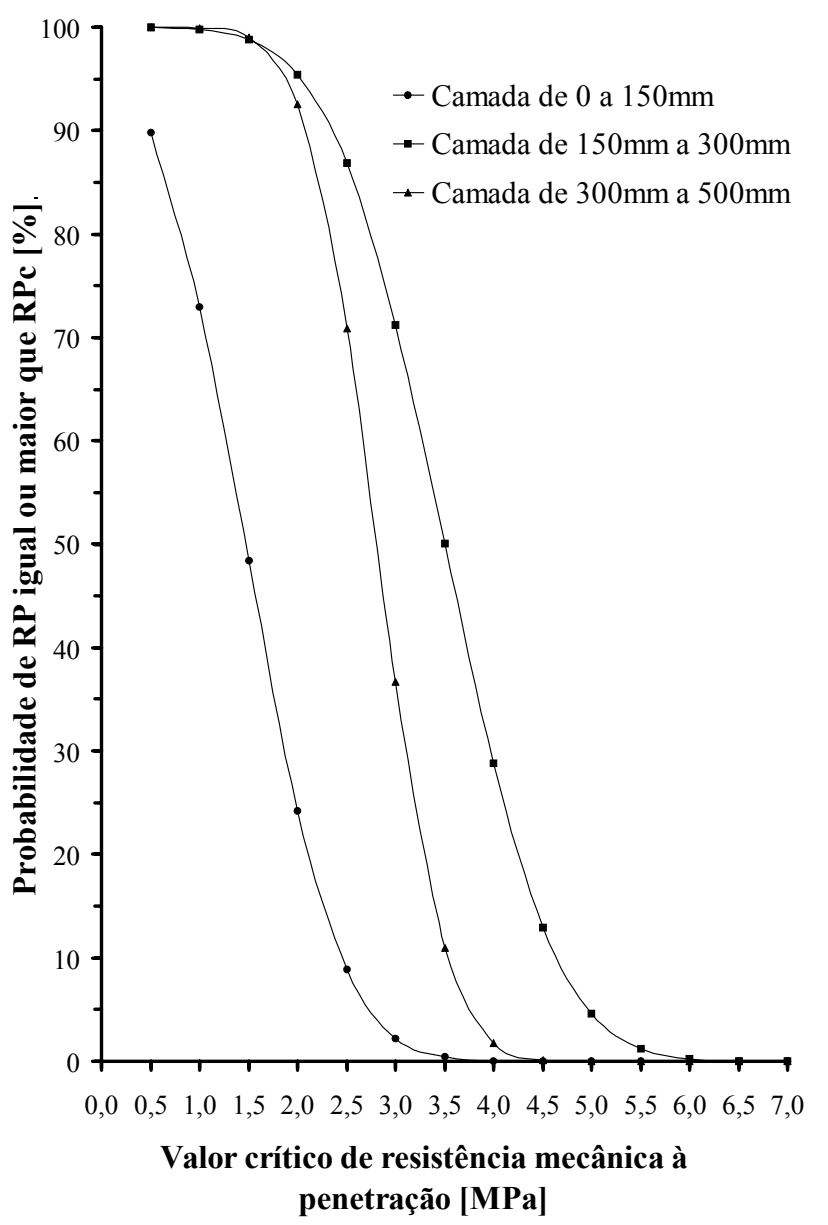

(a)

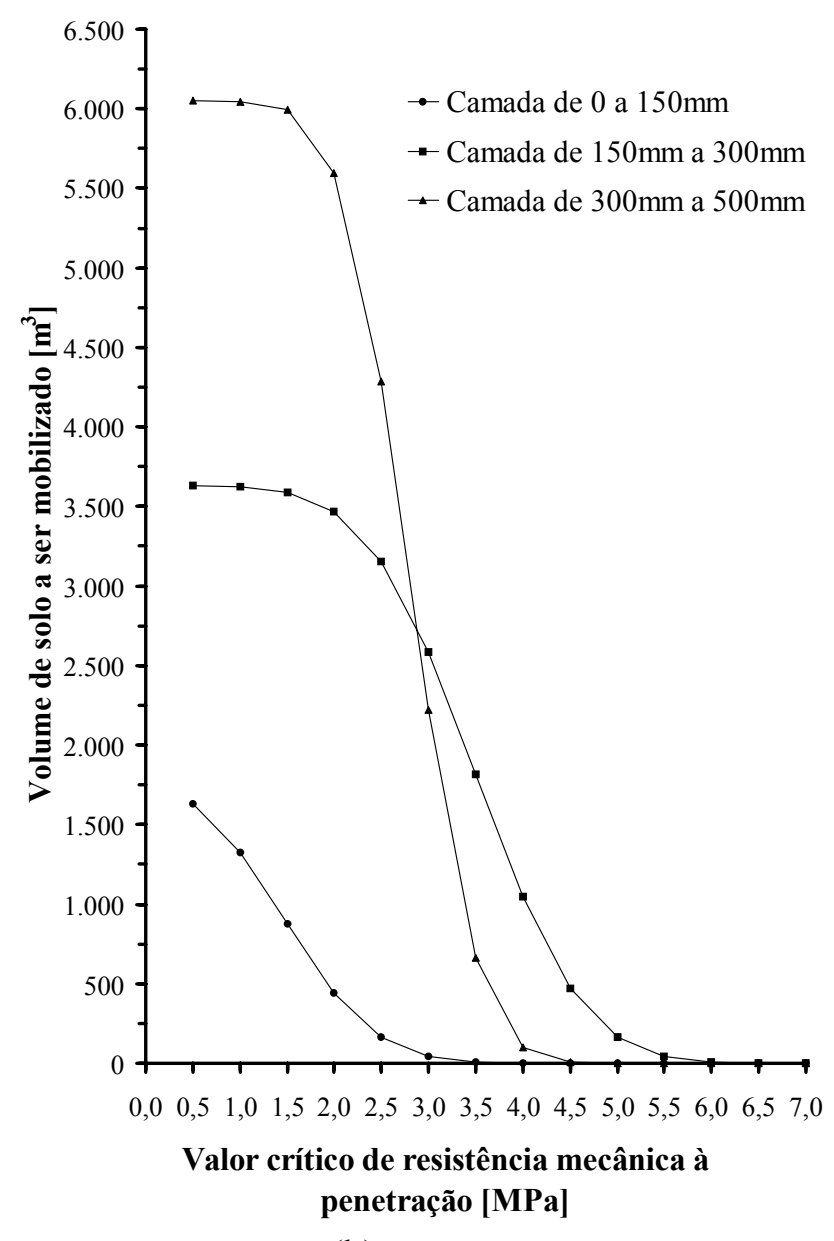

(b)

FIGURA 4. Probabilidade de encontrar valores de RP igual ou superior à RPc (a). Volume de solo a ser mobilizado em função da RPc - método probabilístico (b).

A elevada inclinação do trecho central das curvas de probabilidade mostra que a uma diminuição na resistência mecânica à penetração considerada crítica, corresponde um grande aumento na probabilidade de ocorrência de RP igual ou superior à RPc.

No caso da camada intermediária, diminuindo-se a RPc de 4,5 MPa para 2,5 MPa, há um aumento na probabilidade de ocorrência de RP igual ou superior à RPc, correspondendo a 74 pontos porcentuais (respectivamente, $12,9 \%$ a $86,9 \%$ ).

O estabelecimento do valor crítico da resistência mecânica à penetração e a análise da curva de RP igual ou superior à RPc fornecem subsídios para o diagnóstico da necessidade, ou não, de proceder à operação de subsolagem.

Na Figura 5a, apresenta-se a porcentagem encontrada de pontos experimentais com valores de RP igual ou superior à RPc, nos três perfis horizontais de solo. Na Figura 5b, apresenta-se, para os perfis estudados, o volume de solo a ser mobilizado em função do valor estabelecido como crítico de resistência mecânica à penetração. 


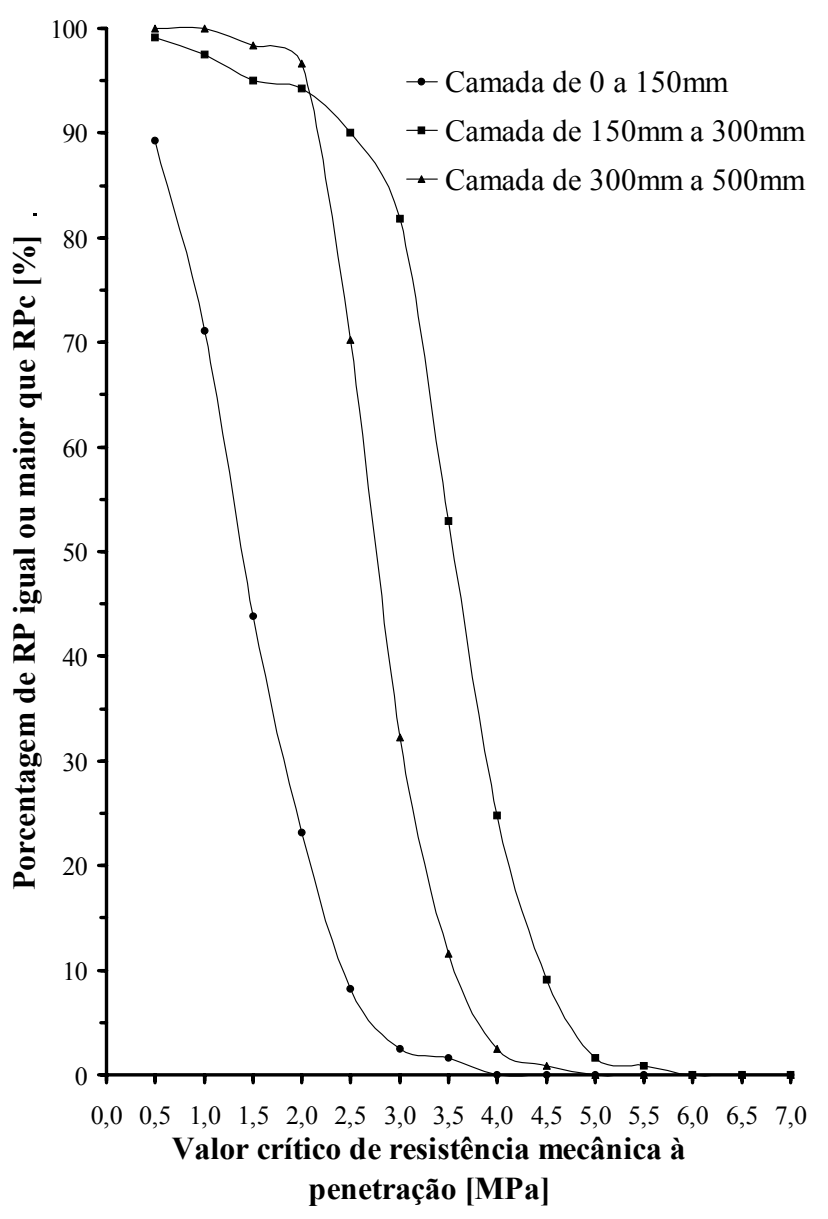

(a)

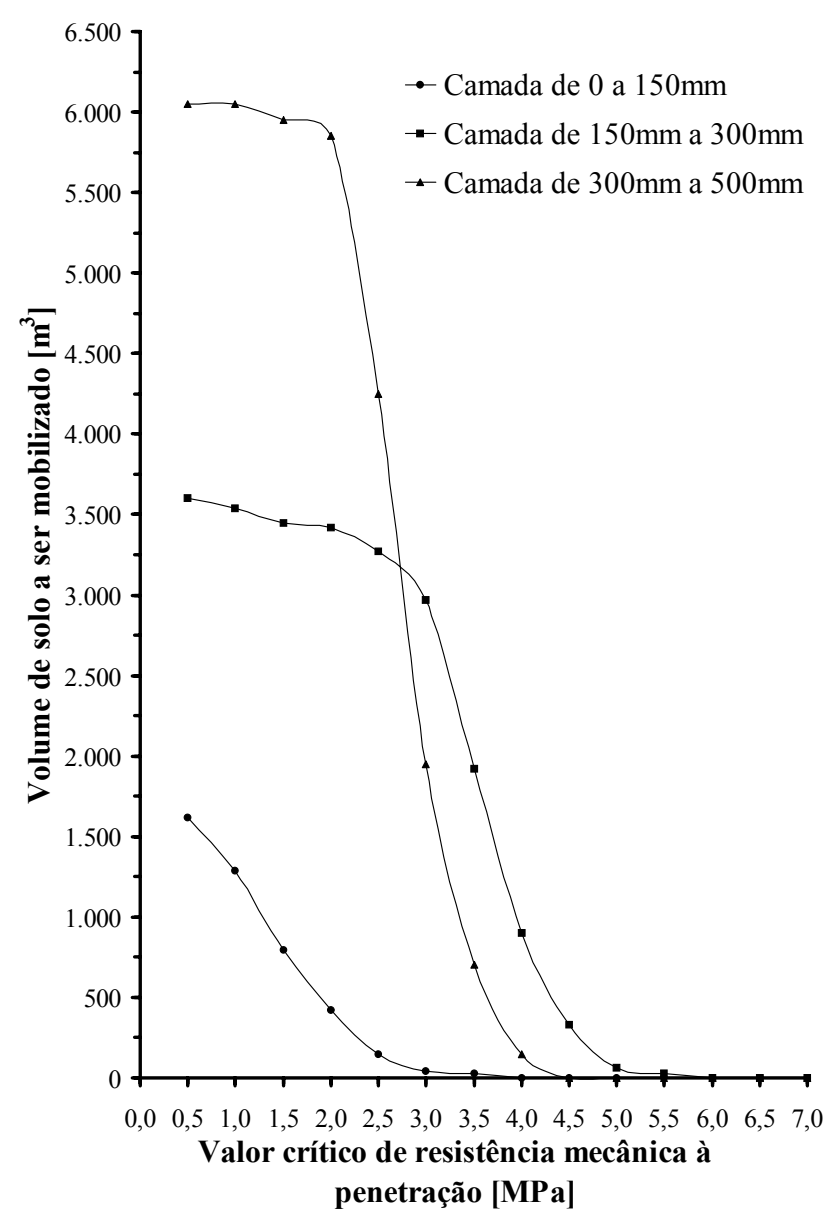

(b)

FIGURA 5. Porcentagem de pontos com RP igual ou superior à RPc nos perfis horizontais de solo (a) e volume de solo a ser mobilizado em função de RPc - método determinístico (b).

Nas Figuras 6, 7 e 8, apresentam-se, no sistema de coordenadas UTM-WGS84, as distribuições espaciais dos pontos com níveis de RP igual ou superior a 2,5 MPa e a 4,5 MPa, para cada camada de solo.

Pode-se verificar que a camada mais superficial possui níveis inferiores de resistência mecânica à penetração em relação às demais (Figura 6). Na Figura 6a, foram poucos os pontos encontrados com $\mathrm{RPc}$ igual ou superior a $2,5 \mathrm{MPa}$ (10 pontos) e que estão dispersos na área estudada. Essa mesma camada de solo não apresentou nenhum ponto com RPc igual ou superior a 4,5 MPa (Figura 6b).

Pode-se verificar que a camada intermediária possui níveis superiores de resistência mecânica à penetração em relação às demais (Figura 7). Na Figura 7a, verifica-se que foram muitos os pontos encontrados com RPc igual ou superior a $2,5 \mathrm{MPa}$ (109 pontos) e que estão presentes em quase toda a área estudada. Já com RPc igual ou superior a 4,5 MPa, foram poucos os pontos encontrados (11 pontos) e estão dispersos na área estudada (Figura 7b).

A camada mais profunda possui níveis intermediários de resistência mecânica à penetração em relação às demais (Figura 8). Na Figura 8a, verifica-se que foram muitos os pontos encontrados com RPc igual ou superior a $2,5 \mathrm{MPa}$ ( 85 pontos) e que estão adensados em regiões por toda a área estudada. Já com RPc igual ou superior a 4,5 MPa, verificou-se um único ponto na área estudada (Figura 8b). 
O método determinístico permite o conhecimento da porcentagem de pontos encontrados com valores de RP igual ou superior à RPc, o volume de solo a ser mobilizado em função de RPc e a distribuição espacial dos pontos com RP igual ou superior à RPc, para qualquer valor de RPc nos perfis horizontais de solo.

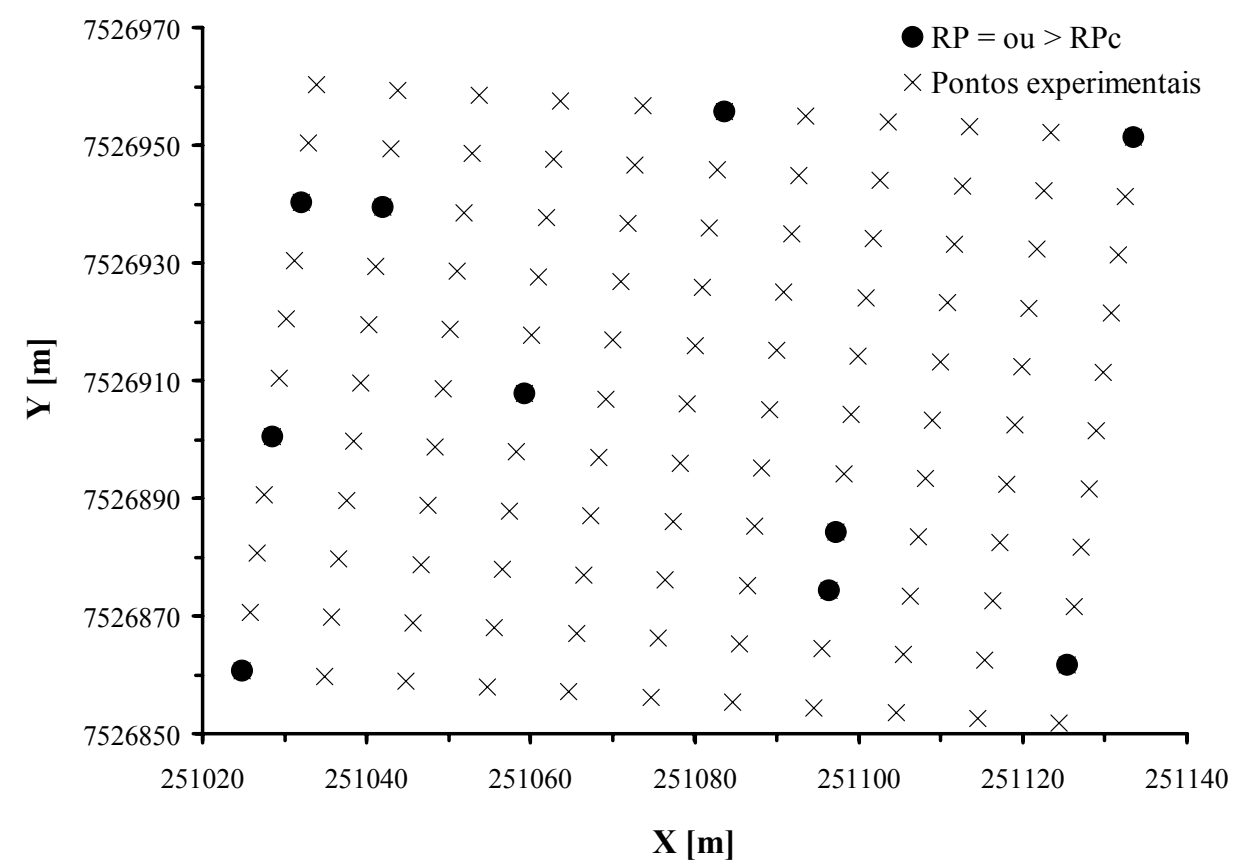

(a)

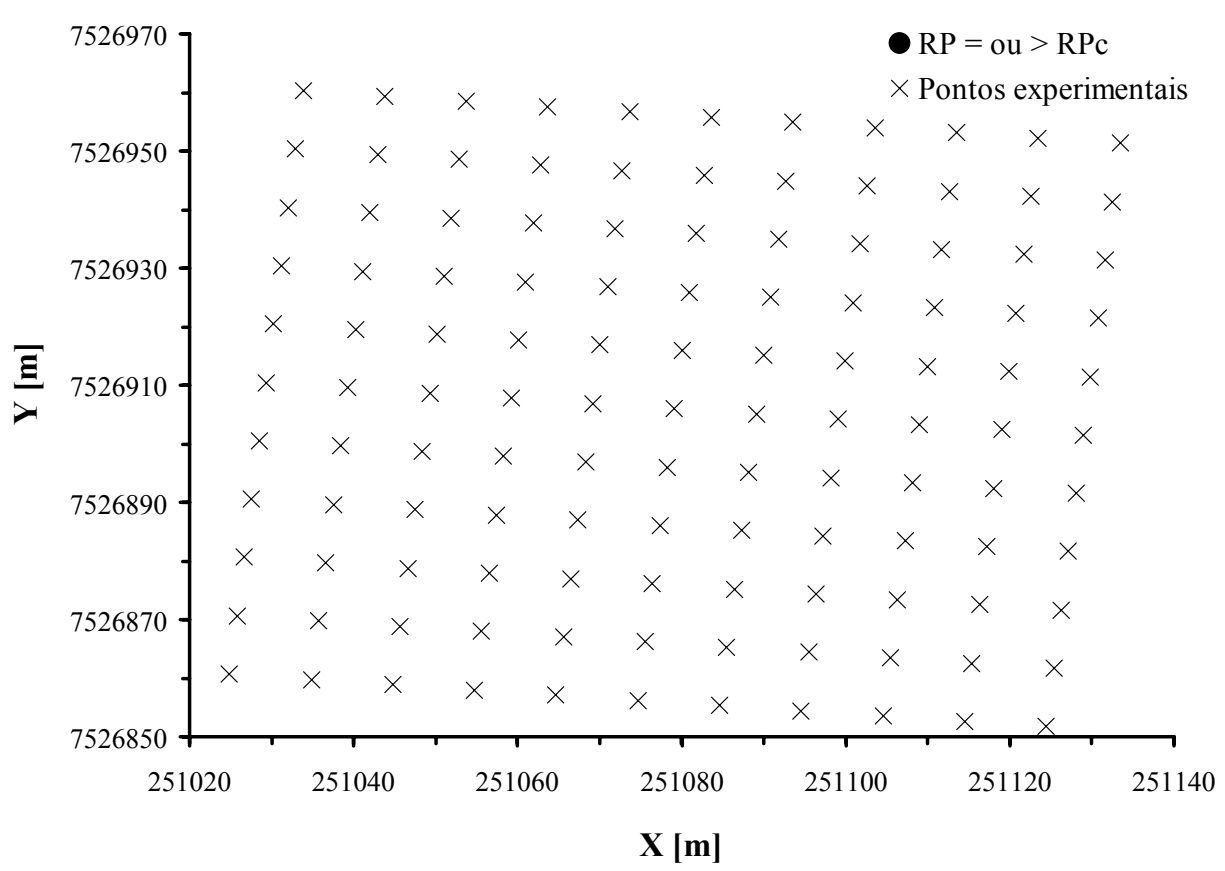

(b)

FIGURA 6. Distribuição espacial dos pontos na camada de solo de 0 a $150 \mathrm{~mm}$. (a) RPc igual ou superior a 2,5 MPa - (b) RPc igual ou superior a 4,5 MPa. 


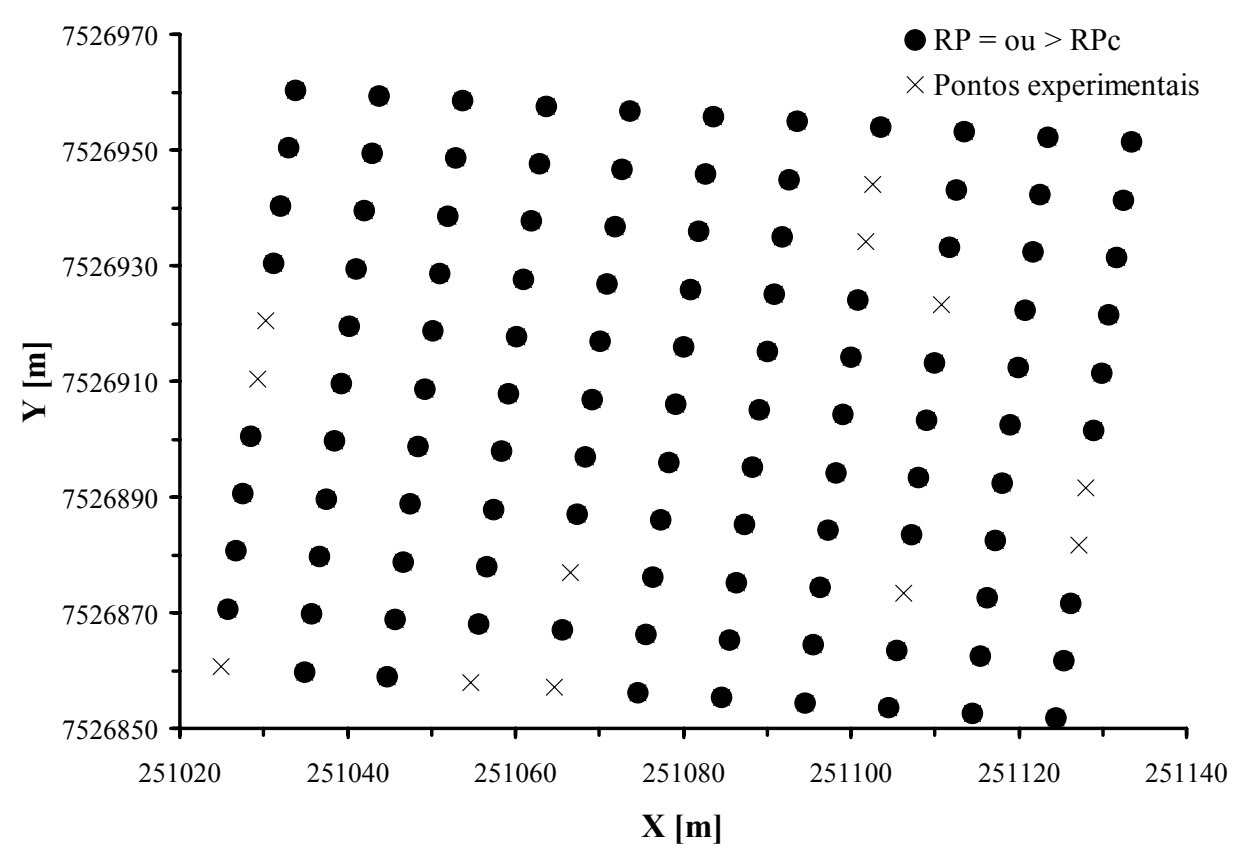

a)

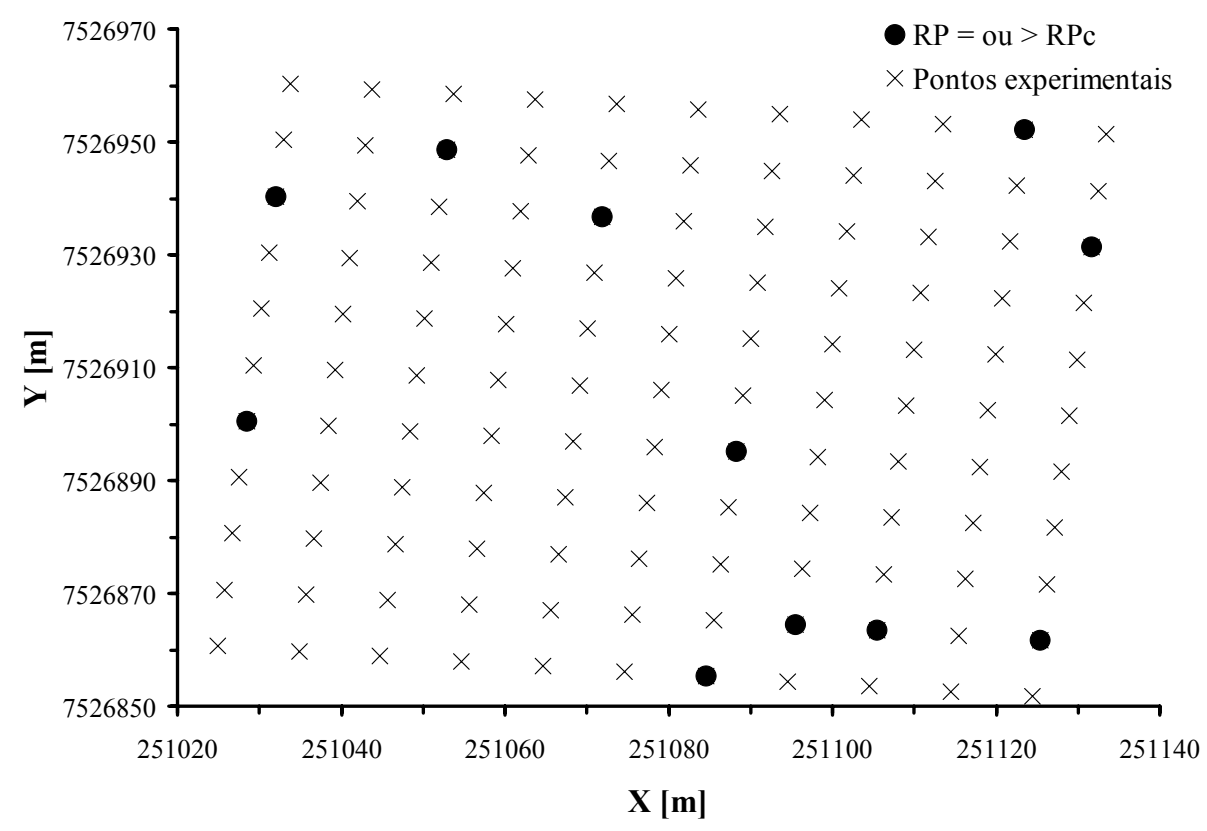

b)

FIGURA 7. Distribuição espacial dos pontos na camada de solo de $150 \mathrm{~mm}$ a $300 \mathrm{~mm}$. (a) RPc igual ou superior a 2,5 $\mathrm{MPa}$ - (b) RPc igual ou superior a 4,5 MPa. 




(a)

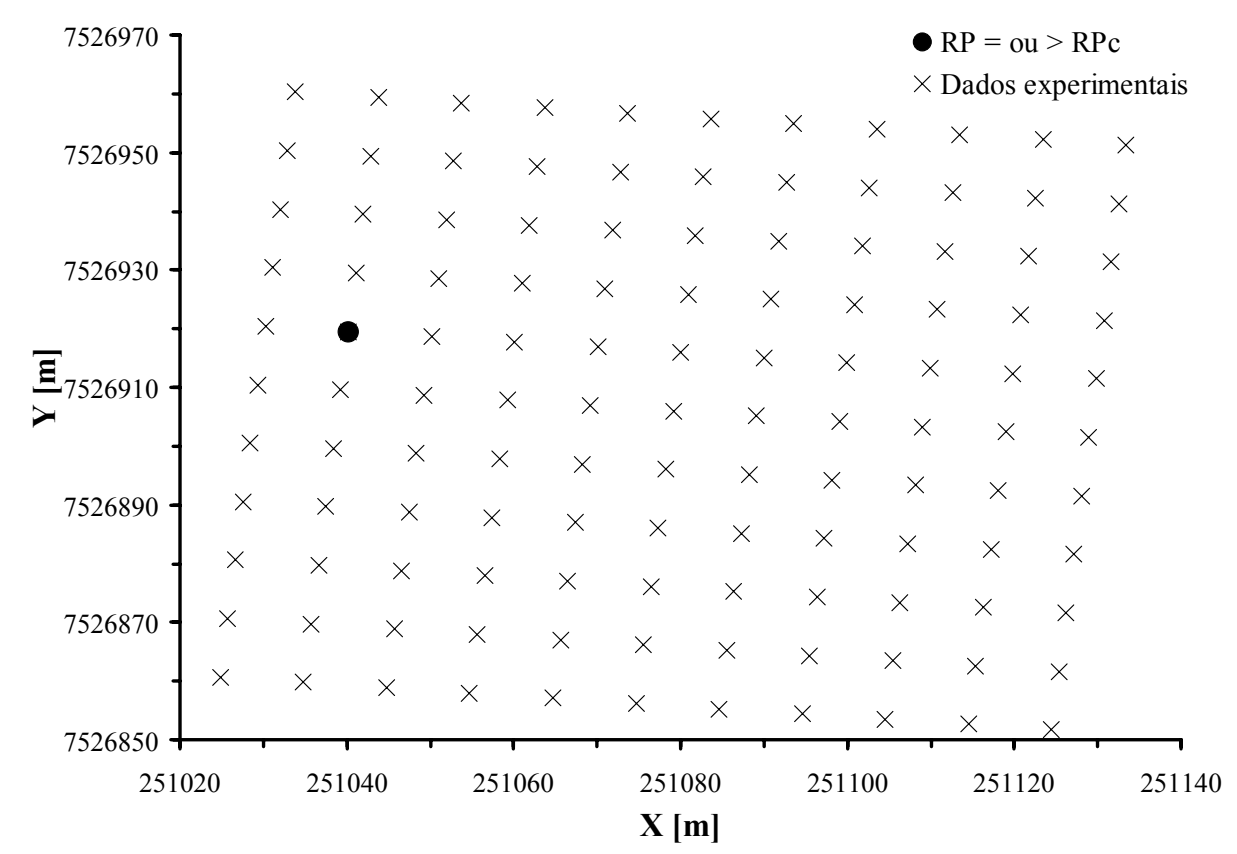

(b)

FIGURA 8. Distribuição espacial dos pontos na camada de solo de $300 \mathrm{~mm}$ a $500 \mathrm{~mm}$. a) RPc igual ou superior a 2,5 $\mathrm{MPa}$ - b) RPc igual ou superior a 4,5 MPa.

\section{CONCLUSÕES}

No método probabilístico, a análise das curvas de probabilidade de RP igual ou superior à RPc e de volume de solo a ser mobilizado fornecem subsídios para o diagnóstico da necessidade, ou não, de proceder à operação de subsolagem. 
O método determinístico permite identificar a porcentagem e a distribuição de pontos encontrados com valores de resistência mecânica à penetração (RP) igual ou superior àquela considerada crítica (RPc) nas camadas horizontais do solo estudado.

No método determinístico, a análise da distribuição espacial de RP, a análise das curvas de porcentagem de RP igual ou superior à RPc e de volume de solo a ser mobilizado fornecem subsídios para o diagnóstico da necessidade, ou não, de proceder à operação de subsolagem.

\section{AGRADECIMENTOS}

Os autores agradecem à Fundação de Amparo à Pesquisa do Estado de São Paulo - FAPESP, por financiar este projeto de pesquisa; à DLG - Automação Industrial e à Usina Santa Lúcia.

\section{REFERÊNCIAS BIBLIOGRÁFICAS}

CAPPELLI, N.L.; UMEZU, C.K.; GUAITOLI, G.; LOZANO, A.F.J. Desenvolvimento de um penetrômetro eletrônico georreferenciado de baixo custo. In: CONGRESSO BRASILEIRO DE ENGENHARIA AGRÍCOLA, 30., 2001, Foz do Iguaçu. Anais... Jaboticabal: Sociedade Brasileira de Engenharia Agrícola, 2001. 1 CD.

EHLERS, W.; KOPKE, F.; HESSE, F.; BOHM, W. Penetration resistance and root growth of oats in tilled and untilled loess soil. Soil and Tillage Research, Amsterdan, v.3, p.261-75, 1983.

EMBRAPA. Centro Nacional de Pesquisa de Solos. Sistema brasileiro de classificação de solos. Rio de Janeiro, 1999. 412 p.

SILVA, G. da. Métodos auxiliares para diagnóstico da necessidade de subsolagem de solos agrícolas. 2002. 137 f. Dissertação (Mestrado em Máquinas Agrícolas) - Faculdade de Engenharia Agrícola, Universidade Estadual de Campinas, Campinas, 2002.

TAYLOR, J.C.; BELTRAME, L.F.S. Por que, quando e como utilizar a subsolagem. Lavoura Arrozeira, Porto Alegre, v.33, n.320, p.34-44, maio/junho 1980.

TORRES, E.; SARAIVA, O.F. Camadas de impedimento do solo em sistemas agrícolas com a soja. Londrina: Embrapa Soja, 1999. 58 p. (Circular Técnica 23) 